\title{
Variations of gamma-ray during thunderstorm by observations in Yakutsk
}

\author{
Anatoliy Toropov ${ }^{1, *}$, Sergey Starodubtsev ${ }^{1}$, Vladimir Kozlov ${ }^{1}$, Juriy Balabin ${ }^{2}$ \\ ${ }^{1}$ Yu.G. Shafer Institute of Cosmophysical Research and Aeronomy SB RAS, Yakutsk, Russia \\ ${ }^{2}$ Polar Geophysical Institute, Apatity, Russia
}

\begin{abstract}
The paper presents the results of a study of the increase in the gamma background in the surface layer of the atmosphere during near thunderstorms in Yakutsk (based on the Cosmic Ray Spectrograph of the IKFIA SB RAS). To register the gamma background, scintillation detectors based on $\mathrm{NaI}(\mathrm{Tl})$ crystals with a size of $63 \mathrm{~mm} \times 63 \mathrm{~mm}$ are used. The range of measured energies is $20-1900 \mathrm{keV}$. The detectors are closed from the sides and bottom with lead $(5 \mathrm{~cm})$ and placed in thermoboxes with a glass window. The detector is located on the roof of the Cosmic Ray Spectrograph building in Yakutsk. Also, synchronous registration of the atmospheric electric field strength was carried out using an atmosphere electric field - mill sensor (with measurement range $+/-50 \mathrm{kV} / \mathrm{m}$ ). The data of continuous recording of an analog signal from a detector during the nearest thunderstorms of 2018 were considered. The analog signal from the gamma detector was fed to the first channel of the E20-10 ADC (L-Card) and recorded continuously on the computer hard disk for one hour (during a thunderstorm) in series of 10 minutes. A signal from an active broadband dipole P-10 antenna ( $0.9 \mathrm{kHz}-900 \mathrm{MHz}$ bandwidth) was fed to the second channel and was also recorded in one file together with the signal from the gamma detector. Synchronous recording of signals from the gamma detector and from the antenna allows accurate timing of gamma photons to electromagnetic signals from nearby lightning. In the studied thunderstorms, based on the results of the analysis of the data obtained, statistically significant variations in the count rate and shapes of the spectrum of gamma - ray at the moment of lightning discharges were found.
\end{abstract}

\section{Introduction}

The appearance of penetrating radiation and the generation of particles in a thunderstorm atmosphere were predicted by various authors at the beginning of the twentieth century $[1,2,3]$. The first reports on the registration of an increase in the neutron flux, the appearance of powerful gamma-ray bursts in the atmosphere during lightning discharges were presented in the 1980s $[4,5]$. These phenomena are currently recorded by

\footnotetext{
* Corresponding author: anatoliy.a.toropov@gmail.com
} 
modern detectors at sea level $[6,7,8,9,10,11,12,13,14]$, at high-mountain scientific stations [15], as well as on spacecraft $[16,17,18,19,20]$.

Numerous studies over the past decades have repeatedly described the strong variations in surface gamma and X-ray emissions associated with thunderstorms. These variations can be divided into two types - short and long. Short and strong bursts of gamma radiation lasting several hundred microseconds (TGF) [6,8] are observed immediately at the moment of a lightning discharge. Long-term increases in gamma radiation (gamma - glow) [14, 20, 21] are observed for seconds and tens of seconds before lightning. The gamma glow often coincides with the transit of thunderclouds.

Another type of prolonged (tens of minutes) increase in gamma radiation is associated with the emission of radon and its decay products $(210 \mathrm{Po}, 210 \mathrm{~Pb}, 214 \mathrm{~Pb}, 214 \mathrm{Bi})[10,23]$ from the ground during heavy rainfall that usually accompany a thunderstorm and is not directly related to lightning or strong electric fields.

Despite the fact that TGF and Gamma-glow differ significantly in their characteristics, it is believed that they are based on one fundamental physical mechanism - relativistic avalanches of runaway electrons [23].

Although a significant number of studies devoted to the observation and theoretical modelling of TGF $[23,24,25,26]$, the nature of their origin is not fully understood.

In this paper, we report the first results of a study of gamma radiation during lightning discharges in submicrosecond data time resolution.

\section{Equipment}

We use a detector based on a $60 * 60 \mathrm{~mm} \mathrm{NaI}(\mathrm{Tl})$ scintillation crystal connected to a photomultiplier tube (PMT). The analog signal from the PMT is amplified and then digitized by a high-speed E2010 L-Card ADC. The range of measured energies is $20 \mathrm{keV}$ 1.2 MeV. An analog electromagnetic signal is fed to the second channel of this ADC, which is received from the near lightning by a precision dipole antenna. The dipole antenna is installed nearby to the scintillation detector and has a receive bandwidth from $0.9 \mathrm{kHz}$ to $900 \mathrm{MHz}$.

Synchronous recording of signals from the gamma detector and from the antenna allows accurate timing of gamma photons to electromagnetic signals from nearby lightning. The sampling frequency of analog signals is $5 \mathrm{MHz}$ for each channel with a 14 bits. Both digitized signals are fed to a data acquisition system based on a personal computer. The data acquisition system is launched directly during a thunderstorm in manual or automatic mode and continuously saves the waveform of the signals from the scintillator and antenna to the hard disk without pauses or any selection of events. The duration of the continuous recording is from 10 to 120 minutes, depending on the data acquisition system settings and the duration of the thunderstorm. The file size for 60 minutes is $67 \mathrm{~GB}$. Further processing of such a file allows you to select the necessary events, view the waveforms with a high time resolution $(0.2 \mu \mathrm{s})$, identify possible interference from electromagnetic interference from lightning, test various methods for programmatically extracting useful signals and avoid difficulties with accurate timing, overflow problems buffer, etc. More details about the design of our detector and the data acquisition technique can be found in [9].

Also, synchronous registration of the atmospheric electric field strength was carried out using an atmosphere electric field - mill sensor (with measurement range $+/-50 \mathrm{kV} / \mathrm{m}$ ).

All detectors are located in an observation post, on the building of a complex of cosmic ray detectors near Yakutsk $\left(61^{\circ} 59^{\prime} \mathrm{N}, 129^{\circ} 41^{\prime} \mathrm{E}, 101\right.$ meters above sea level) and have been in operation since 2017. 


\section{Experimental data and results}

We are considering two thunderstorms that were observed in August 2018 near the observation point.

The first thunderstorm was observed on August 10, 2018 from 02:58 to 05:11 UTC. Figure 1 shows variations in the atmospheric electric field strength (a), low frequency electromagnetic signals from lightning (b) and the count rate of the gamma detector (c), respectively. The data on the graph is presented at one-second resolution. Dashed red lines indicate the start and stop of continuous recording of the scintillator and antenna signals. The duration of the recording was 60 minutes from 03:24:06 to 04:24:06 UTC. In total, 19 nearby lightning strikes were registered during this period. The intensity of the atmospheric electric field reached from $-11.5 \mathrm{kV} / \mathrm{m}$ to $+11 \mathrm{kV} / \mathrm{m}$. The average count rate of gamma radiation pulses is 31 counts per second.

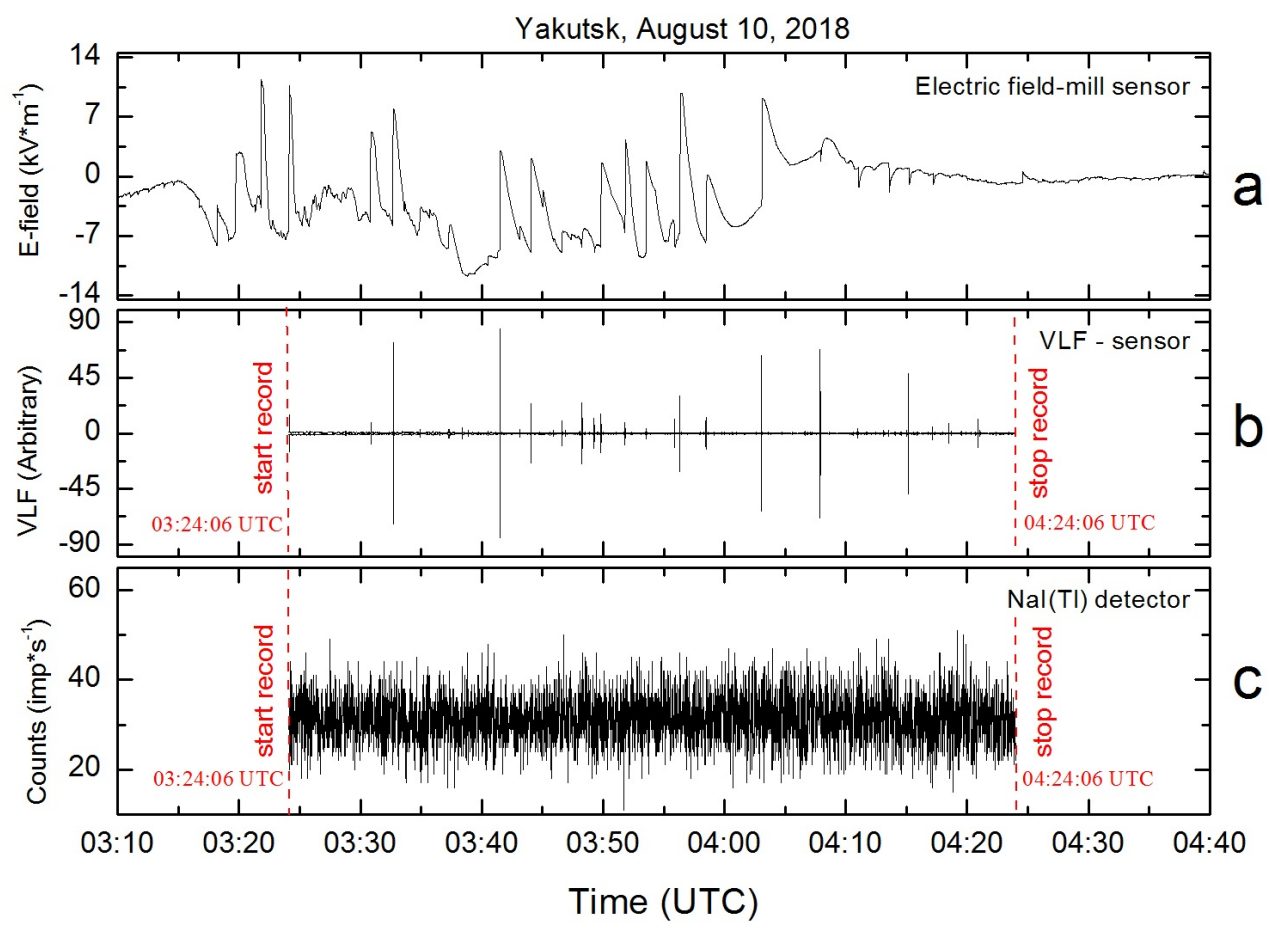

Fig. 1. Thunderstorm event August 10, 2018.

One-sec-binned histories of atmosphere electric field (a). The low-frequency waveform (LF) of the lightning discharge (b). One-sec-binned histories of $\mathrm{NaI}(\mathrm{Tl})$ detectors (c). Red dashed lines show the timing of the start and stop data recording with high time resolution.

The second thunderstorm was observed on August 17, 2018 from 19:23 to 23:57 UTC. Figure 2 shows variations in the atmospheric electric field strength (a), low frequency electromagnetic signals from lightning (b), and the count rate of the gamma detector (c), respectively. The duration of the continuous synchronous recording was, as in the first case, 60 minutes, from 21:18:57 to 22:18:57 UTC.

This thunderstorm was characterized by a high, for this region, intensity of lightning discharges. During the recording session, 124 flashes were recorded, which on average gives a value of one lightning for every 30 seconds. The strength of the atmospheric electric field reached from $-16.4 \mathrm{kV} / \mathrm{m}$ to $+21.5 \mathrm{kV} / \mathrm{m}$. The average count rate of gamma- 
radiation pulses increased monotonically from 20 counts per second up to 29 counts per second.

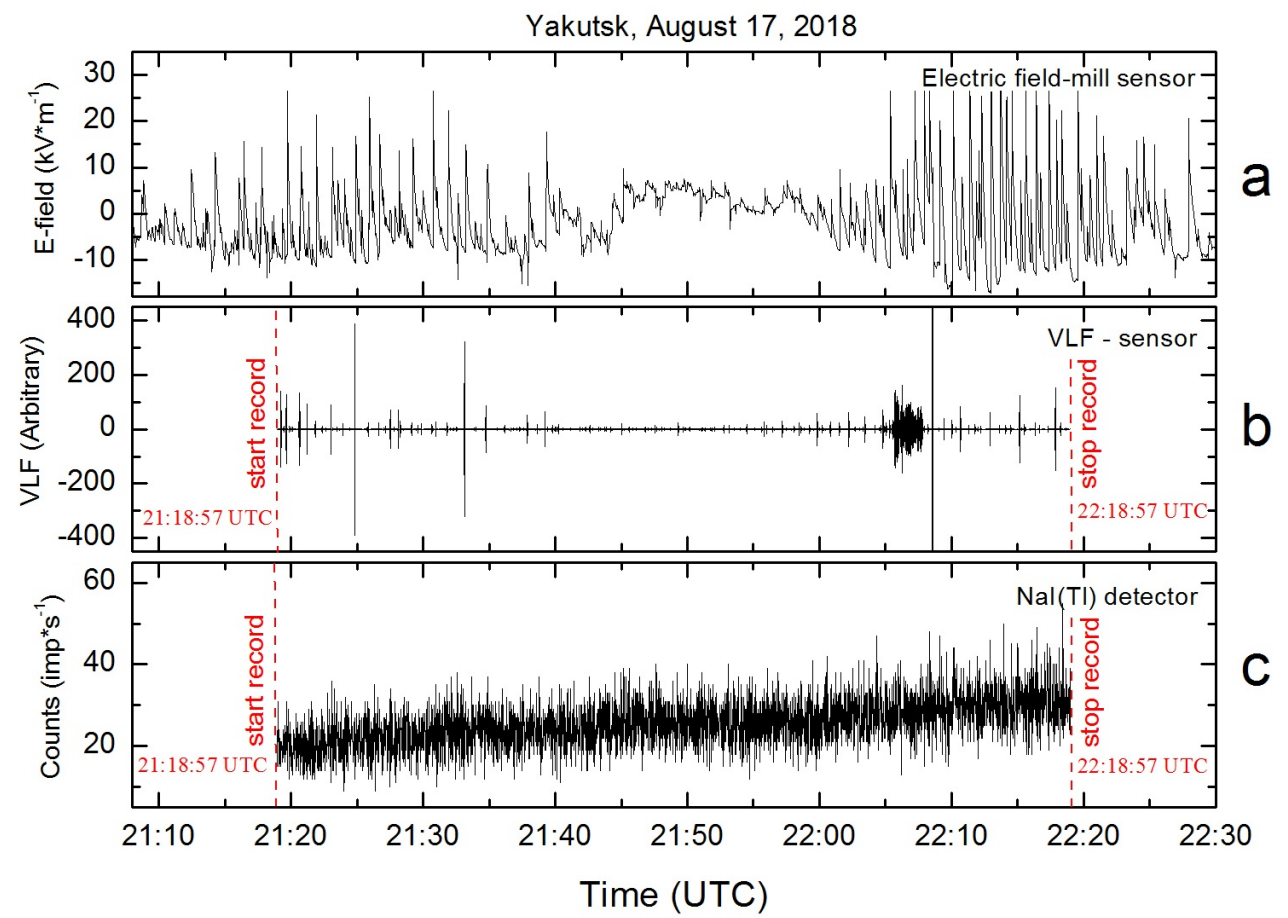

Fig. 2. Thunderstorm event August 17, 2018.

One-sec-binned histories of atmosphere electric field (a). The low-frequency waveform (LF) of the lightning discharge (b). One-sec-binned histories of $\mathrm{NaI}(\mathrm{Tl})$ detectors (c). Red dashed lines show the timing of the start and stop data recording with high time resolution.

It should be noted that in the two thunderstorms we are considering, there are no gamma ray bursts during lightning. However, using our continuous waveform records, we can perform detailed analysis of gamma ray variations in the vicinity of lightning to reveal the fine structure of changes in count rate and spectrum shape. For the analysis, 19 events (lightning) in the thunderstorm on August 10, 2018 and 120 events (lightning) in the thunderstorm on August 17, 2018 were selected (4 lightning strikes were excluded from the analysis due to strong induced interference). A total of 139 events were analyzed.

To find out how the count rate of the gamma detector changes during lightning, six seconds were selected for each of the 139 events. This sample was formed in this way: the first two seconds stood before the lightning, the third second in which the lightning occurred, and three seconds after the lightning. Then, using the epoch superposition method, the total count rate in six seconds was obtained for all 139 events.

Figure 3 shows the result of summing the number of impulses for each of two thunderstorms (a) and for both thunderstorms (b). A noticeable decrease in the count rate of the detector at the moment of lightning and one second after lightning is well observed. In the second after the lightning, a sharp increase in the counting rate is observed. The shapes of the count rate curves in two separate thunderstorms are in good agreement. 


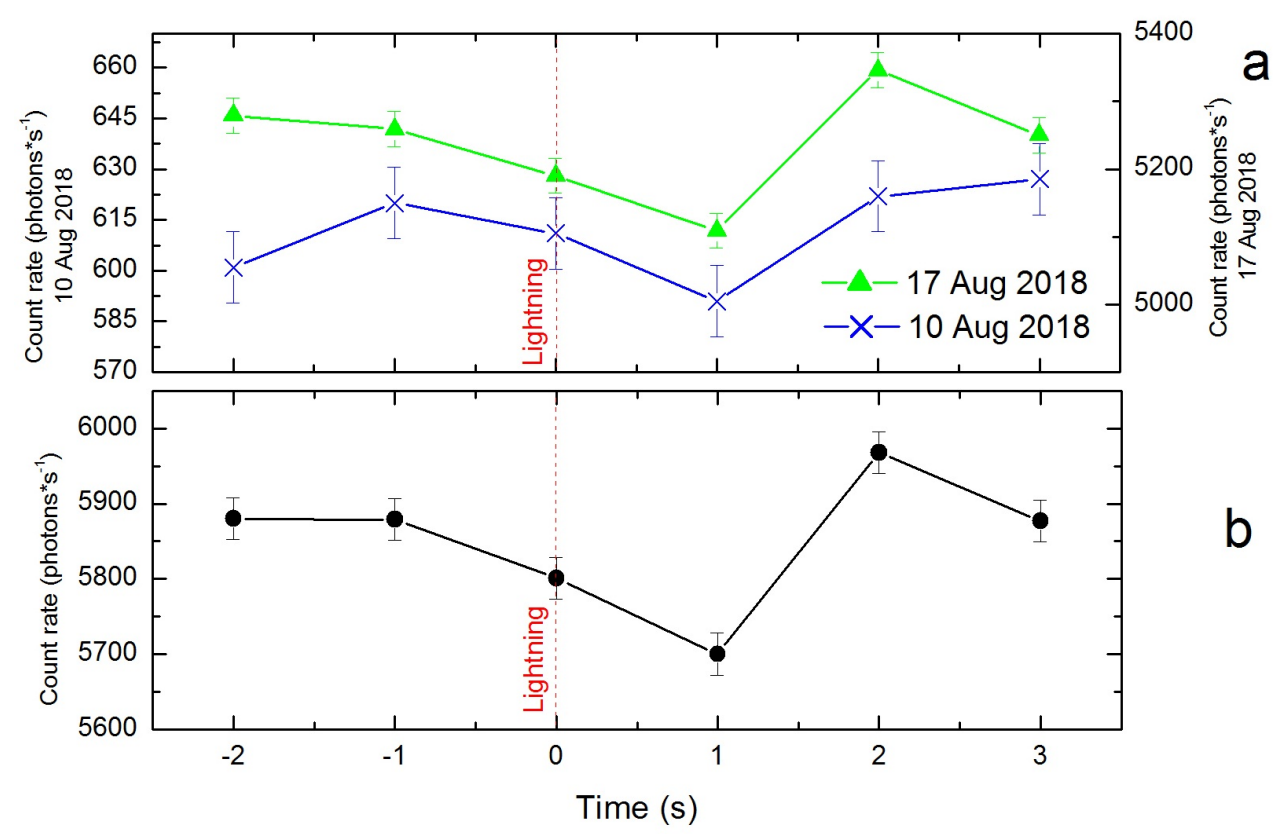

Fig.3. Decrease in count rates of $\mathrm{NaI}(\mathrm{Tl})$ detector at the moment of lightning and after within one second. Counting rate for two thunderstorms (a). Counting rate for two thunderstorms with $\pm 1 \sigma$ statistical errors for an energy range of $0,02-1,2 \mathrm{MeV}(\mathrm{b})$. Red dashed lines show the timing of the lightning discharge.

Of particular interest is information on how the energy spectrum of gamma radiation changed in time before, during and after lightning. We also selected 6-second intervals: the second in which the lightning is fixed, two seconds before the lightning, and three seconds after the lightning. In each second interval, the amplitudes of the pulses recorded by the $\mathrm{NaI}$ (Tl) detector were determined. After that, the amplitudes of the pulses were grouped, each in its own time interval for 139 selected events. As a result, we obtained the energy spectra of gamma radiation for each second in the vicinity of the lightning (for 139 lightning). Figure 4 shows 6 spectra, each of which corresponds to a certain second before and after lightning. 


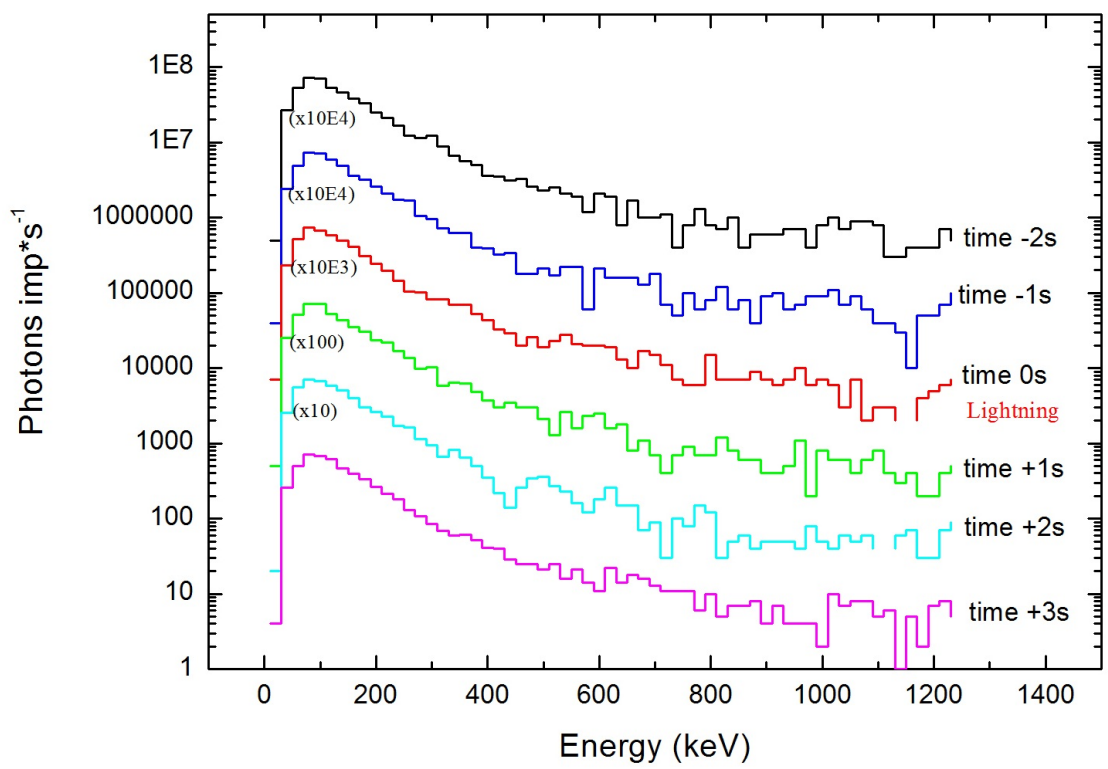

Fig. 4. Comparison between the energy spectra of gamma-ray history in different periods of lightning strike. The spectra in $-2 \mathrm{~s},-1 \mathrm{~s}, 0 \mathrm{~s},+1 \mathrm{~s},+2 \mathrm{~s}$ are shifted by a factor from 10 to $10 \mathrm{E} 5$, respectively, to avoid overlapping.

Significant changes in the spectra in Fig. 4 are manifested at energies above $400 \mathrm{keV}$, and at the first and second seconds after lightning, which may indicate a close relationship with the strength of the atmospheric electric field for which the characteristic period from several to many seconds.
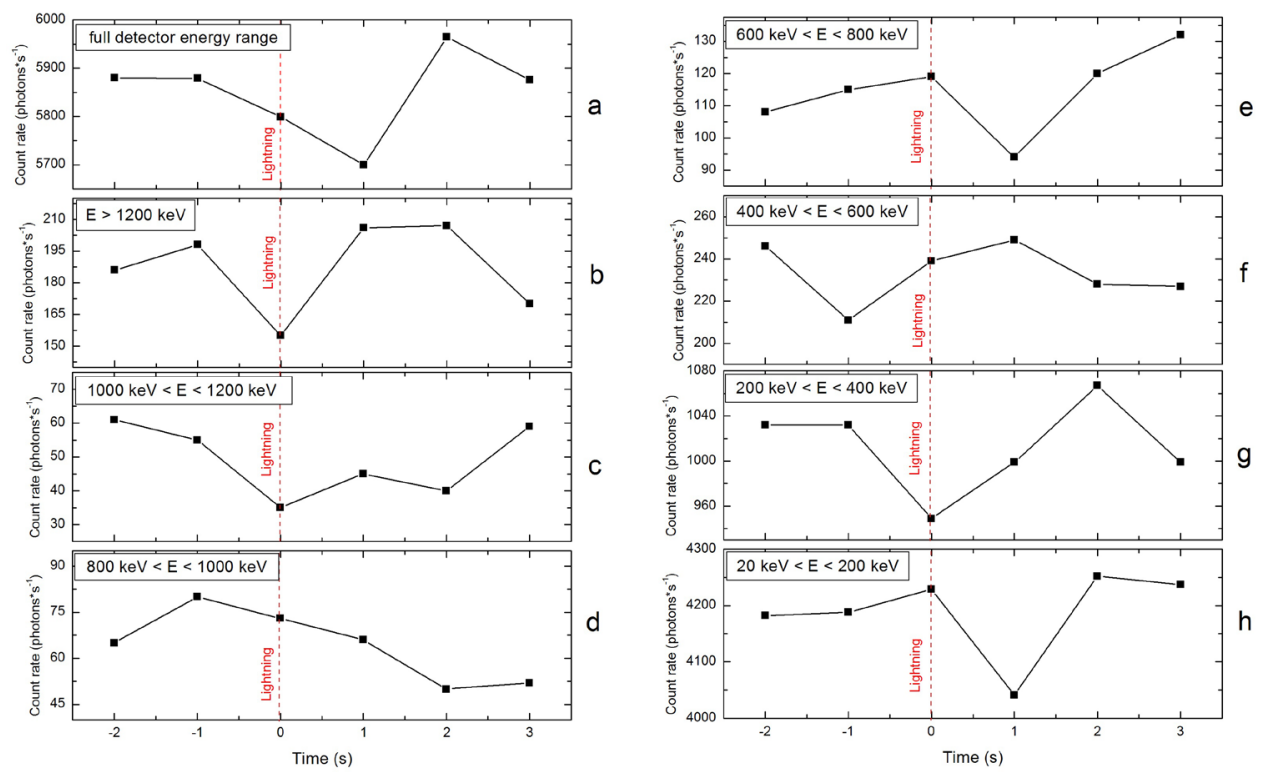

Fig. 5. Variations in the number of impulses in individual energy ranges in time relative to a lightning discharge. Energy range: $0<\mathrm{E}>1.2 \mathrm{MeV}$ (a), $\mathrm{E}>1.2 \mathrm{MeV}$ (b), $1.0 \mathrm{MeV}<\mathrm{E}<1.2 \mathrm{MeV}$ (c), $800 \mathrm{keV}<$ $\mathrm{E}<1000 \mathrm{keV}$ (d), $600 \mathrm{keV}<\mathrm{E}<800 \mathrm{keV}$ (e), $400 \mathrm{keV}<\mathrm{E}<800 \mathrm{keV}$ (f), $200 \mathrm{keV}<\mathrm{E}<400 \mathrm{keV}$ (g), 20 $\mathrm{keV}<\mathrm{E}<200 \mathrm{keV}(\mathrm{h})$. Red dashed lines show the timing of the lightning discharge. 
The graph in Figure 5 shows how the various components of the spectrum have changed over time. The entire length of the spectrum was divided into equal intervals with a width of $200 \mathrm{keV}$. The number of pulses was determined in each interval. Then, for each energy interval, the dependence of the number of impulses before and after lightning was plotted.

It can be seen from the graphs in Figure 5 that the variations of the individual components of the spectrum are complex. However, two things can be noted. First, there is a decrease in the number of impulses at the moment of lightning and per second after lightning in almost the entire energy range. Second: in the energy range $400-600 \mathrm{keV}$, there is an increase in the number of pulses at the moment of lightning and per second after.

\section{Conclusions}

Analysis of gamma-ray variations during two nearby thunderstorms showed that in the gamma ray spectra obtained from 139 individual events (139 lightning), a decrease in the count rate at the moment of lightning and after it was found in the entire energy range from $20 \mathrm{keV}$ to $1.2 \mathrm{MeV}$ except energy range $400-600 \mathrm{keV}$ in which a statistically significant increase is observed.. An increase in the energy range of 400-600 keV probably indicates the generation of positron annihilation radiation $(511 \mathrm{keV})$ from possible photonuclear reactions involving nitrogen, oxygen and gamma-ray inside a thunderstorm atmosphere, as described in [14, 27].

The study was supported by RFBR, research projects No. 18-45-140028 r_a.

The data sets generated and analysed during the current study are available from the corresponding author on request.

\section{References}

1. Wilson, C.T.R., Mathematical Proceedings of the Cambridge Philosophical Society, 22, -1925, pp 534-538. doi:10.1017 / S0305004100003236.

2. Schonland B.F.J., Proc. Roy. Soc. A, 130, -1930, 37-63.

3. Schonland, B.F.J., Proc. Roy. Soc. A, 140, -1933, 314-33

4. Shah, G.N., Razdan H., Bhat G.L., Nature, -1985. V.313. P. 733-755.

5. McCarthy, M. \& Parks, G. K., Geophys. Res. Lett. 12, 393-396 (1985).

6. Dwyer, J. R. et al., Geophys. Res. Lett. 31, https://doi.org/10.1029/2003GL018771 (2004).

7. Toropov, A.A. et. al., J. Atmospheric and Solar-Terrestrial Physics, V. 94, P.13-18, 2013.

8. Paiva G.S., Journal of Applied Physics 105, 2009.

9. Toropov, A.A. et. al., E3S Web of Conferences. Solar-Terrestrial Relations and Physics of Earthquake Precursors. 2018. Doi: 10.1051/e3sconf/20186201013

10. Tran, M. et al., J. Atmos. Sol. Terr. Phys. 136, 86-93 (2015).

11. Bowers, G. S. et al., Geophys. Res. Lett. 44, 10063-10070 (2017).

12. Enoto, T. et al., Nature 551, 481-484 (2017).

13. Smith, D.M.et al. J. Geophys. Res. Atmospheres, https://doi.org/10.1029/2018jd029105 (2018).

14. Wada, Y., Enoto, T., Nakamura, Y. et al., Commun Phys 2, 67 (2019). https://doi.org/10.1038/s42005-019-0168-y 
15. Chilingarian, A. ,Daryan, A., Arakelyan, K., Hovhannisyan, A., Mailyan, B., L. Melkumyan \& Vanyan, Phys. Rev. D, -2010, 82(4), 043009.

16. Drozdov, A. et. al., J. Geophys. Res. 2010.

17. Eack, K.B. et. al., Geophys. Res. Lett. 23, 2915-2918, 1996a.

18. Marisaldi, M. et al., J. Geophys. Res. Space Phys. 115, https://doi.org/10.1029/2009JA014502 (2010).

19. Briggs, M. S. et al., J. Geophys. Res. Space Phys. 115, https://doi.org/10.1029/2009JA015242 (2010).

20. Torii, T., Sugita, T., Kamogawa, M., Watanabe, Y. \& Kusunoki, K., Geophys. Res. Lett. 38, https://doi.org/10.1029/2011GL049731 (2011).

21. Chilingarian, A., Hovsepyan, G. \& Hovhannisyan, A., Phys. Rev. D 83, https://doi.org/10.1103/PhysRevD.83.062001 (2011).

22. Balabin, U.V., et al. Geomgnetism and aeronomy, 3, 376-386, (2014)

23. Gurevich, A.V., Zybin, K.P., Russel-Dupre. R., Phys. Lett. A 165, 463-468, 1992.

24. Dorman, L.I. et. al., J. Geophys. Res. 108 (A5), 1181-1188, 2003.

25. Dwyer, J. R., Geophys. Res. Lett. 30, https://doi.org/10.1029/2003GL017781 (2003).

26. Babich, L. P., JETP Lett. 84, 285-288 (2006).

27. Umemoto, D. et al., Phys. Rev. E 93, https://doi.org/10.1103/PhysRevE.93.021201 (2016). 